\title{
Handedness, parity violation, and the reality of space*
}

\author{
Oliver Pooley \\ Exeter College, University of Oxford
}

19 December 2001; revised 31 July 2002

\section{Introduction}

This paper is about asymmetry rather than symmetry. More specifically, its subject is the sort of spatial asymmetry exemplified by human hands. Hands lack any plane of mirror symmetry. As a result they come in two varieties: left hands and right hands. Similarly we talk of left-handed and right-handed screws, left-handed and right-handed molecules, left-handed and right-handed coordinate systems, or sets of axes, and so on. ${ }^{1}$ Objects of opposite handedness that are otherwise qualitatively identical are "mirror images" of each other. Kant was the first philosopher to see something interesting in such objects. He called them incongruent counterparts. They clearly differ in some way. For example, a glove which might be a perfect fit for a right hand will not fit on its left-handed incongruent counterpart.

A fundamental question with which I shall be concerned is: in virtue of what does one such object differ from its incongruent counterpart; what is the ground of the difference between them? Kant first tackled this question in the context of a philosophical dispute, still very much alive today, that goes by the name of the substantivalist-relationalist debate (Kant, 1992 [1768]). ${ }^{2}$

*To appear in Katherine Brading and Elena Castellani (eds), in preparation, Symmetries in Physics: Philosophical Reflections (Cambridge: Cambridge University Press).

${ }^{1}$ Note that it is not required that the objects lack every sort of spatial symmetry. Screws, for example, can have (discrete) rotational symmetry if their threads are of the correct pitch.

${ }^{2}$ The debate concerns the ontological status of space. Its original protagonists were, on one side, Newton and Samuel Clarke and, on the other side, Leibniz. Substantivalists follow Newton in seeing space (or, in the context of relativistic physics, spacetime) as some kind of substance. It is as real, and as fundamental, as the material objects and events that exist in it. Relationalists follow Leibniz in denying that space is a fundamental entity. They do not deny that material objects are 
I will side with most — although admittedly not all — philosophers in defending an account of incongruent counterparts according to which they are intrinsically identical. ${ }^{3}$ Moreover, I will defend a relational account of handedness according to which the difference between incongruent counterparts is grounded in their relations to each other and to other material objects. Kant thought that there were reasons to reject such an account. Initially he concluded that the difference between left and right hands did indeed come down to a difference in their relational attributes, but that these involved relations to "universal space as a unity" (Kant, 1992 [1768]: 365). Not long after reaching this conclusion, he also rejected this substantivalist account of handedness. Instead he now believed that the difference between incongruent counterparts was fundamentally incomprehensible: that it could only be grasped in perception, through a "pure intuition," and not by any "characteristic marks intelligible to the mind through speech" (Kant, 1992 [1770]: 396).

Accounts of handedness according to which incongruent counterparts are intrinsically identical have recently faced criticism from a new quarter. In World Enough and Space-Time, John Earman argues that the fact that our world displays a lawlike left-right asymmetry poses a serious challenge to what I am calling relational accounts of handedness.

This paper is primarily concerned with the implications of Earman's argument. However, it is useful to consider issues raised by Kant's argument first, for these help isolate the real interpretative difficulties posed by parity violation.

\section{Incongruent Counterparts}

Imagine that you are given a model of a left hand and a perfect mirror-image (i.e. right-handed) duplicate of it. The distance between the tip of the thumb and the index finger will be the same for both hands. Similarly, the angles that the thumbs make to the planes of the palms will be identical in both cases. The two hands are perfectly identical in terms of the distances between their corresponding parts. Kant's way of making this point was to note that a complete description of one hand in terms of the positions of the parts relatively to one another will also be true of its mirror-image (1992 [1768]: 370). For this reason he called them counterparts.

Yet despite this similarity, the two hands are nevertheless incongruent: they

spatially extended. Nor do they deny that material objects stand in determinate distance relations from one another. But they hold that such facts are basic. They deny that space exists apart from such facts; that space has a reality of its own, independent of material objects and their spatial properties.

${ }^{3}$ Harper (1991) and Walker (1978) are two exceptions. 
cannot be made to coincide - they cannot be superposed - by any rigid motion. Kant's own description of the incongruence runs: "the limits of the one cannot also be the limits of the other" (1992 [1768]: 369). A little later he makes the same point by noting that the surface which encloses the physical space of one hand "cannot serve as a boundary to limit the other, no matter how that surface be twisted and turned" (1992 [1768]: 371). It is worth stressing that the relevant notion of possibility here is not that of physical possibility. It is physically impossible to superpose a left hand and its perfect left-handed duplicate if they are both solid material objects. Rather we must abstract from such physical limitations and consider whether it is mathematically possible for the distances between the two objects to be changed continuously in such a way that the two objects eventually coincide. By restricting ourselves to rigid motion, we are only considering changes of the total set of distances that preserve the internal distances between the parts of the two objects.

It is time to state a question that will concern us for much of this section: how is it possible that two objects which are counterparts can nevertheless be incongruent? Answers to this question fall into two categories, within which there are further divisions. One's answer might involve the claim that left- and right-handed varieties of an object differ intrinsically, and then go on to exploit these differences in explaining their incongruence. Within this category one might view these intrinsic differences as primitive and unanalyzable (Kant's later transcendental idealist position is perhaps of this type), or one might view the intrinsic differences as resulting, for example, from a difference in the way the parts of the objects are related to each other. ${ }^{4}$ Alternatively one's answer might involve the claim that incongruent counterparts do not differ intrinsically in any way, and then seek to explain their incongruence in terms of something that is external to each object taken by itself. Kant's earlier substantivalist position is of this type: the difference is to be explained in terms of the objects' different relations to substantival space. But so too is the most economical, purely relational account according to which incongruence is explicable using only the resources of relative distances.

Kant's transcendental idealism aside, the view that left-handed and right-handed objects differ intrinsically has not been popular. There are at least three strong objections to such a view, one of a general nature and two that are specific. The general objection is that it is entirely unclear that we have any conceptual grasp of what such intrinsic differences involve. ${ }^{5}$ In particular, no one has provided an illuminating account of how such intrinsic differences connect with incongruence,

\footnotetext{
${ }^{4}$ Van Cleve calls this latter position "internalism" (Van Cleve, 1991: 22). Clearly such relations must include more than just the relative distances and relations that are reducible to them.

${ }^{5}$ This objection does not tell against Kant's later position, for he was at pains to deny that we have a conceptual understanding of the left-right distinction; it is supposedly only grasped in experience.
} 
or with relevant practical abilities; for example, that I can tell without difficulty of a left hand that it is left-handed and distinguish it from a right hand.

The two specific objections are variations on a theme. ${ }^{6}$ The first exploits the fact that two hands will never be incongruent if embedded in a non-orientable space. ${ }^{7}$ So can hands in such spaces instantiate different intrinsic properties (primitive or otherwise), or not? If they can, one will be able to move a 'left-handed' object into the space occupied by a 'right-handed' one. Does a hand originally exemplifying the property of being 'left-handed' come to lose this basic property and acquire that of being 'right-handed' merely as a result of such a motion? Although they are said to differ in some primitive property, this property appears redundant, making no contact with any other spatial facts about the hands. But if hands in such spaces cannot instantiate the primitive properties of being left- or right-handed, then how is one meant to understand the dependence of whether or not an object can instantiate such a property on the type of space in which it is embedded?

The other objection runs along the same lines, but this time exploits the fact that the incongruence of two hands in part depends on the dimensionality of the space in which they are embedded. One could move a left hand into the space occupied by a right hand if there was an extra spatial dimension through which it could be moved, ${ }^{8}$ just as the letter " $F$ " can be brought into coincidence with its mirror image on the page, $\mathrm{F}$, if one is allowed to lift it off the page. Does whether or not hands have primitive left-handed or right-handed properties depend on the dimensionality of space?

These considerations give us more that enough reason to see whether incongruence can be explained without recourse to the postulation of intrinsic differences. I start by considering the purely relational account. Can we get by merely with relative distances?

The incongruence of left and right hands shows that they differ in some respect. It is surely reasonable to call this difference a purely spatial difference. That left and right hands match in terms of the distances between their parts shows that this difference not grounded in these distances. However, the relationalist is not committed to view that every spatial difference between two objects supervenes on a difference in the spatial arrangement of their parts. He can also appeal to

\footnotetext{
${ }^{6}$ The following type of considerations are emphasized by Nerlich (1994: 51-3), but see also Frederick (1991: 8) and Van Cleve (1991: 22-3).

${ }^{7}$ Or, to adopt relationalist language, if the spatial relations between them are such that the lowest-dimensional spaces in which they are embeddable are all non-orientable. Some believe that failure to provide an account of the orientability of space might show that relationalism is ultimately untenable. I disagree, for reasons elaborated on below, pp. $6 \mathrm{ff}$.

${ }^{8}$ Although string theorists would have us believe there are in fact six or more such dimensions, they are 'too small' to permit the required motion.
} 
the distance relations that hold between the two objects, and between them and other objects. Once this is acknowledged, there would seem to be no reason why the relationalist cannot view the incongruence of two counterparts as grounded in such external relations.

Now nothing in my original definition of incongruence precluded relationalism. ${ }^{9}$ In fact, one might even think that incongruence has been defined in purely relational terms: rigid motion is defined in terms of the constancy of the distances between the parts of an object and coincidence (the occupancy of the same boundaries) is defined in terms of the distances between (the corresponding parts of) the two objects. Even when we seek the ground of such incongruence, it seems that the relationalist has no reason to be embarrassed. It is simply a mathematical fact, and a comprehensible one, that, when constrained to obey the algebraic relationships of Euclidean geometry, some numbers (the possible distances between two congruent counterparts) can be continuously altered so as to vanish while others (the distances between two incongruent counterparts) cannot.

The claim that the relationalist can not only accommodate but can explain incongruence is significant. As already noted, Kant came to the view that the incongruence of counterparts was, in a certain sense, fundamentally incomprehensible. He thought that it could be grasped only in experience. In his Inaugural Dissertation he writes:

between solid bodies which are perfectly similar and equal but incongruent, such as left and right hands (in so far as they are conceived only according to their extension), or spherical triangles from two opposite hemispheres, there is a difference, in virtue of which it is impossible that the limits of their extension should coincide - and that, in spite of the fact that, in respect of everything which may be expressed by means of characteristic marks intelligible to the mind through speech, they could be substituted for one another. It is, therefore, clear that in these cases the difference, namely the incongruity, can only be apprehended by a certain pure intuition. (Kant, 1992 [1770]: 396)

But this is simply a non sequitur. Suppose that incongruent counterparts are intrinsically identical; that they do not differ in themselves in any way. So a fortiori we cannot, restricting ourselves to just the internal distances between the parts of two hands, understand or explain the hands' incongruence. Nevertheless we can both understand and explain their incongruence in terms of the different ways any two hands can be related to each other. It just does not follow from the fact that we

\footnotetext{
${ }^{9}$ The definition deliberately does not follow others that can be found in the literature; see e.g. Earman (1989: Chap. 7), Brighouse (1999) and Huggett (2000).
} 
cannot intellectually grasp an intrinsic difference between left and right hands that we can have no intellectual grasp of the basis of their incongruence, or that this incongruence is manifest only in experience.

Those whose intuitions lead them still to side with Kant at this point need to respond to the following challenge: Suppose, for the sake of argument, that the relationalist is correct in asserting that all spatial facts are reducible to facts about relative distances between material objects. The relationalist will insist that any two coexisting hands stand in some quite determinate distance relations from one another. How can it be denied that the possibility of incongruent counterparts is already secured? ${ }^{10}$ When things are put this way, surely the burden of proof is now on someone who wishes to assert that the incongruence or otherwise of the two hands is not determined despite the various facts about the distances between the them.

So far we have seen that relative distances alone are sufficient to ground the incongruence of two handed objects that are otherwise identical. However, there are two other questions about handedness that have exercised philosophers. The first is: in virtue of what is an object handed? The second is: what accounts for the particular handedness of a handed object — what makes it, say, a left hand?

My initial characterization of a handed object suggested that its key feature was that it lacked any plane of mirror symmetry. This is, again, a feature highlighted by Kant, who noted that a handed object cannot consist of "two halves which are symmetrically arranged relatively to a single intersecting plane" (Kant, 1992 [1768]: 370). One might think that this is a characteristic that is reducible to facts concerning the relative distances between the parts of the object. ${ }^{11}$ However, as Nerlich notes, more is needed.

He defines an enantiomorph as follows:

each reflective mapping of [an enantiomorph] differs in its outcome from every rigid motion of it. (Nerlich, 1994: 51)

Otherwise the object is a homomorph. Nerlich's principal contention is that "whether a hand... is enantiomorphic or homomorphic depends on the nature of the space it is in. In particular it depends on the dimensionality or the orientability, but in any case on some aspect of the overall connectedness or topology of the space" (1994: 53). Nerlich's claim, then, is that an object's being handed is not reducible

\footnotetext{
${ }^{10}$ I have been tacitly assuming that the relative distances involved are those of an infinite $\mathcal{N}$ dimensional Euclidean space. Things are obviously more complicated when one considers more general sets of relative distances. For some discussion, see Pooley (2002: Chap. 6).

${ }^{11}$ Carol Brighouse, however, worries that talk of a plane of symmetry and of lines intersecting it at right-angles is not obviously relationally acceptable (Brighouse, 1999: 56-8). The relationalist strategy that I outline below sidesteps Brighouse's worries.
} 
to facts about the relative distances between material objects. It also depends on the dimensionality and orientability of the space in which the object is embedded.

Even if one were to agree with Nerlich about this, it is not clear that this observation can be used as an argument against a relational account of space, and for two reasons. The first is that we should ask why the relationalist about space is under any obligation to offer an equivalent, relationally pure definition of enantiomorphy. He believes that spatial facts are exhausted by the catalogue of relative distances between material points (and the fact that these must obey certain constraints). We have seen that this is enough to allow for the possibility of incongruent counterparts. If it turns out that substantivalism underwrites properties, such as enantiomorphy, which are not well-defined by the lights of the relationalist's ontology, then so much the worse for enantiomorphy. Nothing in our experience of objects such as hands forces us to admit the existence of such additional properties, just as (so the relationalist would like to maintain) nothing in our theorizing about motion forces us to admit the reality of space.

Secondly, it has yet to be shown that the relationalist cannot provide a definition of enantiomorphy. Nerlich's observations might suggest that the relationalist needs to come up with a surrogate definition of orientability, and this is indeed the strategy that most have pursued (Brighouse, 1999; Huggett, 2000). Unfortunately for the relationalist, it has not been entirely successful. For example, although an object's being multiply related is a necessary condition of its being embeddable in a non-orientable space (for all non-orientable spaces are multiply connected), it is not a sufficient condition. Kant's own example of triangles on a sphere is precisely an example of multiply related yet enantiomorphic figures. Huggett suggests that what is needed is a "general representation theorem of the form 'space $S$ is orientable iff relations of type are instantiated' " (Huggett, 2000: 225). No such theorem has been forthcoming. The relationalist also needs an account of the dimensionality of space.

I wish to propose that the relationalist has a way of sidestepping some of these difficulties. First, in order to be able to exploit (rather than explain) the fact ${ }^{12}$ that the exact nature of an object's multiple relatedness (if it is multiply related) can determine whether or not the spaces in which it is embeddable are orientable or not, the relationalist does not need to have the type of representation theorem to which Huggett alludes. Second, as Huggett notes, the relationalist can talk freely of embedding the particular relative distances between the parts of some material object in a space, so long as the operation is understood to be a purely

\footnotetext{
${ }^{12}$ If it is a fact. This is something that the relationalist will want to prove. However, the mere possibility that it is a fact is enough, at this stage of the dialectic, to save the relationalist. The onus is now on the substantivalist to prove that the exact nature of an object's multiple relatedness does not fix the orientability of the lowest-dimensional embedding spaces. Thanks to Jeremy Butterfield and Carl Hoefer for saving me from overstating the relationalist's case.
} 
mathematical exercise (Huggett, 2000: 224).

So now suppose, additionally, that the relationalist has an account of the dimensionality of space. ${ }^{13} \mathrm{He}$ can then define the enantiomorphy of a material object by adopting Nerlich's definition, but now with respect to all abstract embedding spaces of the specified dimension. For example, if it is the case that according to an empirically adequate relational theory space has three dimensions, the relationalist can claim that an object is an enantiomorph iff, with respect to every possible abstract 3-dimensional embedding space, each reflective mapping of the object differs in its outcome from every rigid motion of it. On this definition, planar objects count as homomorphs as do 3-dimensional hands that are multiply related so as to be embeddable only within non-orientable 3-dimensional spaces. Hands that are embeddable only within orientable 3-dimensional spaces count as enantiomorphs, even though they are, of course, embeddable in spaces of higher dimensions.

One suspects that Nerlich himself would be prepared to grant much, if not all of this (see, e.g., Nerlich, 1994: 61-2). He would still see the phenomena of handedness and enantiomorphy as supporting substantivalism for two reasons. One, which takes us beyond our topic, is that he already sees the relationalist's employment of brute, unmediated, spatial relations as suspect (see, especially, Nerlich, 1994: 23-33). The second is that he believes that the substantivalist account of enantiomorphy is simply more illuminating. The relationalist account, to the extent that it explains anything at all, does so on the back of the substantivalist one:

... the orientability of space does determine the handedness of hands, for it determines which paths there are in a space which a hand might take. It is a genuinely explanatory idea. Spatial relations, I suggest, explain enantiomorphy only by way of entailing the orientability of the containing space, and it is through that understanding that we come to grasp handedness. (Nerlich, 1994: 67)

This, however, is disputable on two grounds. First the relationalist account of incongruence (if not of Nerlich's definition of enantiomorphy) does not appeal to facts about embedding spaces. All it deals in are facts about relative distances and about what changes of relative distances are possible, and "possible" here need not be thought of as constrained by an embedding space.

Second, the facts that the substantivalist appeals to are really of exactly the same type. Ultimately the spatial relations between the parts of space are either

\footnotetext{
${ }^{13}$ This might be fixed by the adoption of some specific relational dynamical theory. Relational theories typically simply assert, via the choice of some relative configuration space for example, that the relative distances between material objects are constrained to be embeddable in, say, a Euclidean space of no more than three dimensions; see, for example, Barbour and Bertotti (1982). Note that this is also likely to fix the orientability of space directly.
} 
simply assumed to be, or are stipulated to be, constrained in certain ways. That the substantivalist believes that relations between material points, and the possible motions of those points, are mediated and constrained in virtue of those points being located at various spatial points is, quite simply, a distraction. How is Nerlich to explain the incongruence of two hand-shaped regions of space? Space and its shape might be easier to picture than algebraic facts about distances, but the idea that it is more explanatory is illusory. For even in this case, the facts that ultimately explain are precisely algebraic facts about distances.

I now return to the second question raised on page 6: what accounts for the particular handedness of a handed object? To see that, once again, nothing more than relative distances is required, it will prove useful to consider the anti-relationalist argument of Kant's 1768 paper.

In this paper, Kant explicitly characterizes his aim as that of providing a "clear proof that: Absolute space, independently of the existence of all matter. . has a reality of its own" (1992 [1768]: 366). In other words he sets out to vindicate Newton's substantivalist conception of space over Leibniz's relationalist conception. ${ }^{14}$ His argument does not challenge the claim that the relationalist can account for the incongruence of left and right hands. Rather it suggests that the difference between left and right goes beyond the relational facts so far cited.

After rehearsing the various definitional facts about incongruent counterparts and after noting that their incongruence cannot be grounded in a difference in how their parts are related, Kant makes the following claim:

... imagine that the first created thing was a human hand. That human hand would have to be either a right hand or a left hand. The action of the creative cause in producing the one would have of necessity to be different from the action of the creative cause producing the counterpart.

Kant rightly notes that this is incompatible with relationalism:

\footnotetext{
${ }^{14}$ Sometime prior to 1768 , Kant is generally acknowledged to have held a Leibnizian, relational view of space. This, however, is a matter of some controversy amongst Kant scholars. Some argue that he is better seen as advocating some kind of compatibilism. Things are further complicated by the fact that, as already discussed, just two years after apparently arguing for a Newtonian view of space, Kant published his first "critical" work, the Inaugural Dissertation of 1770, is which he rejects both substantivalism and relationalism, arguing instead that space is in some sense 'in us', a form of our intuition. The seeds of Kant's transcendental idealism about space are already discernible in the 1768 essay. However, the extent to which incongruent counterparts by themselves led Kant to transcendental idealism is again a matter of some controversy.

What should be stressed for the purposes of the present discussion is that it is evident that by 1768 Kant believed that his argument from incongruent counterparts provided a decisive reason to reject a purely relational account of handedness. Nowhere in his subsequent writings does Kant retreat from this claim.
} 
... there is no difference in the relation of the parts of the hand to each other, and that is so whether it be a right hand or a left hand; it would therefore follow that the hand would be completely indeterminate in respect of such a property. In other words, the hand would fit equally well on either side of the human body; but that is impossible. (1992 [1768]: 371).

How should the relationalist respond to this particular challenge? He can simply deny Kant's initial premise, that every hand in an otherwise empty universe is necessarily either a right or a left hand. Certainly Kant is wrong to suppose that the lone hand's being of indeterminate handedness entails the absurdity that it can fit on both sides of a human body. For suppose that one is given a relational description of a hand and also a relational description of a handless human body that has various internal asymmetries involving the heart and other organs. One might then ask on which side of this body does the hand (properly) fit: the side on which the heart is, or on the other side?

The relationalist certainly should not answer "both". Rather he will deny that the question makes sense independently of a specification of the relative distances between the body and the hand. There are two incompatible ways in which a body satisfying the relational description and the lone hand could coexist in a single universe. According to one such way, the hand will fit on the side of the body that the heart is on. According to the other way, the situation is reversed; the hand fits on the other side of the body to that on which the heart is. But either way, the hand will fit determinately on one, and only one, side of the body. And which side it fits is determined by the distances between the hand and the various parts of the body, i.e. by purely relational facts.

Although the relationalist's contention that the difference between left and right hand supervenes on the distances between them and between other material objects does not entail a patent absurdity, one might still wonder whether it is not in tension with our evident ability to recognize, for example, left hands as left. However, a little reflection suggests that the account the relationalist must give of our practical abilities and linguistic practices - of how we teach the meanings of "left" and "right" and of the fact that we are often prone to confuse left and right - is far more plausible than any account which postulates our recognizing an intrinsic difference between incongruent counterparts or recognizing that the hand bears some relation to (invisible) space. In fact, so far as I know, no one has attempted to give a genuine account of our abilities that postulates our recognizing either of these things.

The basic elements of a relational account have been outlined many times. ${ }^{15}$ We have seen that, despite holding that left and right hands are intrinsically iden-

\footnotetext{
${ }^{15}$ See, for example, Earman (1989: Ch. 7, §2), Gardner (1990: Ch. 17), Huggett (1999: 209-12),
} 
tical, the relationalist will also acknowledge that they fall into two equivalence classes defined, roughly speaking, by the relation of congruence. But it is then straightforward to understand how a practice of distinguishing members of these classes might involve all the hands of one class being given one 'name' ("left"), and all hands of the other being given another ("right"). Causal links between the speakers who are party to this practice, and between the speakers and actual hands, will ensure that the practice remains consistent. Together with an ability to recognize a hand as congruent or incongruent to hands with which one has previously been presented and has been told are left or right, these causal links are all that are required.

According to the relationalist account, therefore, the only facts about a left hand that make it left, is the fact that we call it "left", that it is congruent to every other hand that we in fact call "left" and incongruent to every hand that we call "right". ${ }^{16}$ Such an account of the meanings of "left" and "right" is, of course, very close to a causal theory of reference for proper names. And in certain respects the terms "left" and "right" are very much like names. What was it about Immanuel Kant, for example, that made it correct for his contemporaries to call him "Immanuel Kant"? Nothing, other than the fact that he was actually known as "Immanuel Kant", that there was a practice of calling him "Immanuel Kant" and so on.

Can this really be all there is to the left-right distinction? I believe that it is. Such a point of view receives indirect support from what Jonathan Bennett calls the Kantian Hypothesis (Bennett, 1970). ${ }^{17}$ This is the claim that chiral terms such as "left" and "right" cannot ultimately be explained without ostensively demonstrating, for example, a left hand. Various chiral terms can be explained in terms of each other. For example, one can define "left" in terms of "clockwise" and other, related, notions. But to break out of a rather tight circle, one must ultimately show what one means by "clockwise" or by "left." Non-chiral words will never be enough.

This thesis can be put in the form of a predicament that Martin Gardner calls the Ozma problem (Gardner, 1990: Chap. 18). Suppose that we are in radio contact with some extra-galactic civilization. Gardner's Ozma problem is: "Is there any way to communicate the meaning of "left" by a language transmitted in

Hoefer (2000: $\S 3$ ) and Saunders (2000: $§ 3.3)$. These accounts, of course, differ from each other, and from my own, in minor ways.

${ }^{16}$ The question "in virtue of what is a left hand left" is thus rather misleading. I should perhaps stress that my favoured relational account of handedness is not part of a general nominalism according to which the instances of any general term " $\mathrm{X}$ " have in common only the fact that we call them all "X". It is only the left-right contrast, not handedness per se, that is purely nominal.

${ }^{17}$ The reason for attributing this hypothesis to Kant is Kant's insistence, noted earlier, that the difference between left and right cannot be made intelligible through concepts. 
the form of pulsating signals? By the terms of the problem we may say anything we please to our listeners, ask them to perform any experiment whatever, with one proviso: There is to be no asymmetric object or structure that we and they can observe in common" (1990: 167). If Bennett's 'Kantian hypothesis' is correct, we cannot manage the task without some asymmetric observable object in common with our alien friends. Appealing to the side of the body on which the heart is won't help, for example, because the alien hearts, if they have hearts, might be on the right. ${ }^{18}$ If the mechanism in virtue of which the terms "left" and "right" refer is indeed what I have suggested it is, the difficulty of explaining their meanings within the constraints of the Ozma problem are readily understandable.

Although Kant concludes in favour of substantivalism in his 1768 essay, he appears to do so very much by default. There is no explanation in his essay of how substantival space is able to ground that which relationalism supposedly cannot: the incongruence of counterparts. If substantivalism and relationalism represent two genuinely exhaustive alternatives, then an argument against one would be an argument for the other. But as I noted earlier, Kant quickly came to the view that they are not jointly exhaustive, and instead opted for the tertium quid of transcendental idealism.

However, Hoefer (2000) has recently pointed out that there is one way in which the postulation of substantival space can be used to secure Kant's intuition that a hand in an otherwise empty universe is necessarily either a left or a right hand. One is to imagine that the universe contains a single hand and that the space in which the hand exists is the substantival space of our actual world. One would then appear to be able to appeal to facts of the following sort: in the imagined possible world, the lone hand is either determinately congruent to the hand-shaped region of space that is actually and currently occupied by my left hand, or it is determinately congruent to the hand-shaped region of space that is actually and currently occupied by my right hand. In the first case the lone hand is left-handed, in the second it is right-handed ( $c f$. Hoefer, 2000: 241). I wish to make five observations about this substantivalist account of a lone hand's determine handedness.

First, as Hoefer is keen to stress, such an account is only open to the substantivalist who believes that there are primitive facts about which points of space or spacetime in two different possible worlds count as the "same" point. In terms

\footnotetext{
${ }^{18}$ Actually, as will become apparent, we have known since the 1950s that we could exploit the fact that the laws of nature violate parity. One might worry that, since we are communicating through photons, we cannot rule out the possibility that our alien correspondents live in an antimatter galaxy. Were the laws CP invariant, their following our instructions and carrying out an experiment illustrating parity violation would lead them to conclude that "left" meant right. Fortunately we can appeal to CP violating experiments to overcome this potential problem. Moreover TCP invariance is not a problem because our communicating at all presupposes that we agree about "before" and "after"!
} 
that will be more familiar to philosophers, it is not enough that one be a substantivalist; to give such an account, one must also be a haecceitist. Since the issue was brought into focus by Earman and Norton's version of Einstein's "hole argument", many philosophers have concluded that commitment to such primitive identities, and the corresponding haecceitistic differences between possible worlds, is not an obvious concomitant of a belief in the fundamental reality of space or spacetime (see, especially, Brighouse, 1994; Rynasiewicz, 1994; Hoefer, 1996). ${ }^{19}$

Second, the account only works for possible worlds the space(time)s of which have the same global topology as that of the actual world. This is because it is not clear what transworld identity relations, primitive or otherwise, could hold between two non-diffeomorphic spaces.

In fact, that the two spaces have the same global topology is not even sufficient. Let us assume substantivalism and primitive transworld identity for the sake of argument. There will nevertheless be spacetime points that are the location of some instantaneous stage of my left hand in this world but that form a perfect sphere in some other possible world. All that is required is that the region they constitute is topologically identical to my left hand. The handedness of the hands of this possible world will thus be undetermined for they will all be equally (in)congruent to the space actually occupied by my left hand. ${ }^{20}$

Third, although these complications do not tell against Hoefer's reconstruction being faithful to Kant's thinking - Kant and his contemporaries implicitly assumed that the spaces of all possible worlds were isometric to $E^{3}$ - the reconstruction certainly does not do justice to Kant's assertion that having a particular handedness is a matter of having the correct relation to space as a unity. According to Hoefer's Kant, it consists in having the right relation to particular regions of space, for example, the region which is actually the location of my left hand. This is something that Kant denies (Kant, 1992 [1768]: 365). ${ }^{21}$

Fourth, it is evident that the account is surprisingly close to the relationalist account of handedness just given. In particular, note how the account 'explains' what it is to be a left-handed hand-shaped region of space. This is held to be merely a matter of congruence to the actual material hands that we in fact, actually,

\footnotetext{
${ }^{19}$ I should also mention that not all philosophers agree. Belot and Earman, for example, argue against substantivalists who reject haecceitism, whom they brand "sophisticated substantivalists" (Belot and Earman, 2000, 2001). For a response to their arguments, see Pooley (2002: Chap. 9).

${ }^{20}$ the restriction to points underlying an instantaneous stage of $m y$ left hand is incidental. There are possible worlds in which the spacetime points forming the worldtubes of every actual hand form worldtubes of perfect spheres, or of objects whose handedness changes over time etc.

${ }^{21}$ Hoefer does point out that "no particular points, lines, rays or regions [of space] are the ones that have to be mentioned" (2000: 243). However, this hardly makes it the case that being of a particular handedness is a matter of a hand's relation to space as a unity, rather than, say, to space as a plurality. Kant is explicit in his denial that handedness involves a hand's relation to places (and hence, presumably, to sets of these).
} 
call "left". If one believes in primitive identity, one can exploit the fact that a particular hand-shaped region of space exists in a large class of possible worlds to secure the handedness of material hands in all these world. One is effectively securing a vicarious congruence between material hands in two different possible worlds by way of particular hand-shaped regions of space that are supposed to exist in both. If this is all there is to the substantivalist's explanation of the handedness of the hands in one-hand worlds, then the relationalist's assertion that such hands do not have a determinate handedness starts to look decidedly less exceptionable.

The final, and related, observation is that the account surely does not connect with our epistemological situation. We certainly do not recognize hands as left in virtue of recognizing their congruence to particular regions of space. Such regions are invisible. This underlines the fact that the account is effectively a marriage of a relational account of handedness with substantivalism and haecceitism, so as to secure the determinate handedness of hands in other possible worlds.

\section{The challenge of parity violation}

The conclusion of the first part of this paper is that objects of opposite handedness that are otherwise identical, such as idealized left and right hands, do not differ intrinsically in any way and, furthermore, that their opposite handedness is a matter of their external spatial relations to each other (and to a language using community that has assigned quite arbitrary labels to the two incongruent classes of such objects). Recently, philosophers have realized that modern physics appears to suggest that there is a substantial difficulty with this view. The problem goes beyond the mere existence of incongruent counterparts; it arises from parity violation, the fact that the laws of nature appear to distinguish between left and right.

The transformation of parity inversion is spatial reflection through the origin. In the context of quantum field theory, it is closely connected to two other discrete transformations, namely time reversal (temporal reflection through the origin) and charge conjugation (the interchange of matter and anti-matter). If space has an odd number of dimensions (as the space of our world has) parity inversion maps a handed object onto an incongruent counterpart. ${ }^{22}$ If the parity transformation is a symmetry of a theory, it will always map physically possible states of affairs onto physically possible states of affairs. Hence if a particular handed object or process is physically possible, then parity conservation implies that its incongruent

\footnotetext{
${ }^{22}$ Consider the Cartesian coordinates of a left hand relative to an arbitrary (left- or right-handed) set of axes. Now consider a passive parity inversion: a point that originally had coordinates $(x, y, z)$ is assigned new coordinates $(-x,-y,-z)$. The coordinates of the left hand with respect to the new coordinate system are the coordinates of a possible right hand with respect to the original coordinate system.
} 
counterpart will also be physically possible. Conversely, if parity inversion fails to be a symmetry, then there will be at least some cases where it maps a physically possible state of affairs onto one that the law prohibits. If we assume that spatial translations and rotations are symmetries of the theory, then these will be cases where there is a type of enantiomorphic object or process of one handedness that is physically possible and yet its incongruent counterpart is not. ${ }^{23}$

John Earman (1989: Chap. 7) was the first to suggest that, while the relational account outlined above may be able to deal with incongruent counterparts, the fact that a law of nature violates parity poses a more recalcitrant problem. In fact, he sees parity violation as having implications for substantivalist-relationalist debate in much the way Kant initially thought that incongruent counterparts had. The reason is that he believes the substantivalist can ground the left-right asymmetry exhibited in processes governed by parity-violating laws whereas the relationalist cannot. I shall shortly question this assumption.

Earman's example of a process that exemplifies such a law involves the decay of neutral hyperons that was experimentally investigated by Crawford et al. (1957) as a test for parity violation. An example that may be more familiar is the $\beta$-decay of radioactive cobalt atoms, the subject of the first experimental confirmation of parity violation (Wu et al., 1957). In such a decay the electron and its antineutrino are preferentially emitted along the axis of nuclear spin. Given this, there are two, mirror-image possibilities, depicted in Figure 1. In (a) the electron is emitted in the same direction as the spin of the cobalt nucleus, in (b) the electron is emitted in the opposite direction.
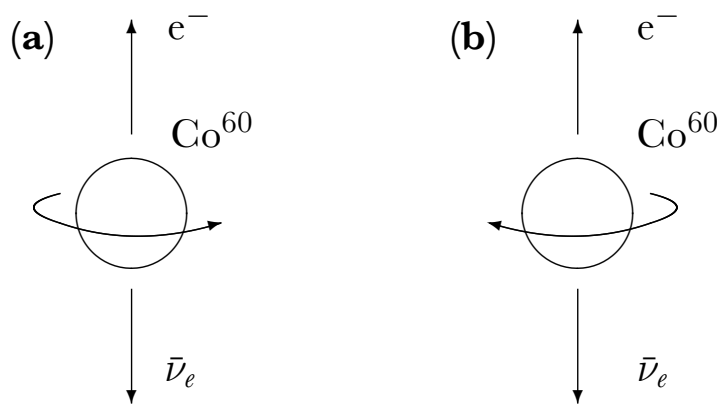

Figure 1: $\mathrm{Co}^{60} \rightarrow \mathrm{Ni}^{60}+e^{-}+\bar{\nu}_{e}$

The weak interaction, which governs this decay process, fails to be symmetric

\footnotetext{
${ }^{23}$ Actually, this is what is required of a deterministic parity-violating law. If the law is essentially probabilistic, then parity will also fail to be a symmetry if different probabilities are assigned to a pair of counterpart yet incongruent processes.
} 
under parity inversion. The decay (a), it turns out, is much more probable than (b). In Wu's experiment, a sample of cobalt 60 was cooled to near absolute zero and then subjected to a magnetic field to align the nuclear spins: many more electrons were detected emerging in the direction of nuclear spin than in the opposite direction.

In terms of this example, here is how Earman puts the challenge to the relational account of handedness defended above:

The failure of mirror image reflection to be a symmetry of laws of nature is an embarrassment for the relationist account sketched... for as it stands that account does not have the analytical resources for expressing the law-like asymmetry for the analogue of Kant's hand standing alone. Putting some 20th century words into Kant's mouth, let it be imagined that the first created process is a $\left[\mathrm{Co}^{60} \rightarrow \mathrm{Ni}^{60}+\right.$ $\left.e^{-}+\bar{\nu}_{e}\right]$ decay. The absolutist has no problem in writing laws in which $[(\mathrm{a})]$ is more probable than [(b)], but the relationist... certainly does since for him $[(\mathrm{a})]$ and $[(\mathrm{b})]$ are supposed to be merely different modes of presentation of the same relational model. Evidently, to accommodate the new physics, relational models must be more variegated than initially thought. (1989: 148)

Without doubt, Earman has put his finger on something. But one might wonder whether that the full scale of the problem has been stated. Two things are worth saying immediately. First, given the conclusion of the first part of this paper, is it obvious that the absolutist (a.k.a. the substantivalist) has no problem "writing laws in which (a) is more probable than (b)"?

Modulo the qualifications made above, Hoefer's haecceitist substantivalist can secure the handedness of lone hands in otherwise empty possible worlds. In particular, Hoefer's substantivalist can claim that the reality of space grounds the genuine distinctness of a world in which the first created process perfectly resembles (a) and a world in which it perfectly resembles (b). However, he does so by claiming that processes of type (a) stand in different relations to particular bits of space to those in which processes of type (b) stand. It seems doubtful that such a substantivalist will want to write relations to particular bits of space into the laws. As Hoefer says: "It seems wrong for a law of nature to contain reference to a particular, contingent physical object. But it seems (to me) at least as wrong for a law of nature to contain reference to particular bits of space..." (Hoefer, 2000: 253).

Second, Earman's way of setting up the challenge, in terms of the "first created process", suggests a relationalist response that echoes Herman Weyl's response to Kant's argument. Weyl wrote, "Had God, rather than making first a left hand and then a right hand, started with a right hand and then formed another right hand, he would have changed the plan of the universe not in the first but in the second 
act, by bringing forth a hand which was equally rather than oppositely oriented to the first created specimen" (Weyl, 1952: 21). Similarly, perhaps the relationalist can maintain that whether the first created process is a typical decay governed by the weak interaction, or whether it is a possible but atypical decay, will depend on its incongruence or otherwise to the majority of subsequent similar decays. This, I think, is ultimately what the relationalist has to say. One aim of the rest of this paper is to highlight some of the costs involved.

The Weyl-style relationalist is obviously allowed the relationally acceptable distinction between a world where the first decay process is typical (i.e., congruent to the majority of subsequent decays) and one where it is atypical. He is also allowed the distinction between (parity violating) worlds where the majority of decays are handed in the same way and (parity symmetric) worlds in which decays of opposite handedness occur with equal frequency. The fundamental problem faced by any account of handedness according to which incongruent counterparts do not differ intrinsically is, in those worlds where the majority of decays are handed in the same way, how can this asymmetry be explained? If the decay modes (a) and (b) are intrinsically identical, what could ground their different likelihoods?

The challenge posed by parity violation is thus well-put by Van Cleve, who anticipates Hoefer's unease with laws that make reference to particulars:

God could no doubt see to it that certain kinds of particles always decay into configurations of the same handedness. But we need to be able to suppose that the result in question comes about through law rather than divine supervision. How can it be law that particles always... display decay modes of one orientation rather than another, if orientation is not intrinsic? If one particle has decayed in left-handed fashion, how does the next particle 'know' that it should do likewise? It's instruction cannot be to trace a pattern of a certain intrinsic description; it can only be to do what the first particle did.

The problem here is not 'action at a distance', though perhaps that will trouble some. It is rather that the required laws would make ineliminable reference to particular things, whereas it is generally supposed to be of the essence of laws that they state relations of kind to kind. (Van Cleve, 1991: 21-2)

In a moment I shall suggest that in one respect Van Cleve is wrong; the problem is action at a distance and not ineliminable reference to particular things. But to see how reference to particular things - whether they be particle decays or regions of space - can be avoided, we need to review some of the details of the law that describes parity-violating processes. 

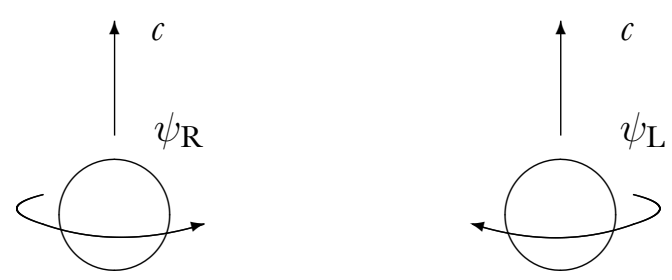

Figure 2: chirality in the zero mass limit

\section{A relational account of parity violation}

That Nature treats left- and right-handed varieties of certain processes differently is puzzling. And yet we have an extremely well-confirmed physical theory describing how it does so. How does the mathematics of the theory work? Does it do so by revealing that left-handed and right-handed varieties of handed objects differ intrinsically after all?

The most fundamental description of parity-violating interactions so far formulated is that given by the Weinberg-Salam gauge field theory, part of the Standard Model. ${ }^{24}$ The Weinberg-Salam theory treats elementary particles, such as electrons and quarks, as excitations of Dirac quantum fields interacting via gauge boson fields (the photon, the $W^{+}$and $W^{-}$, and the $Z$ ). Parity violation results from the fact that the theory treats the 'left' and 'right' chiral components of the same Dirac field quite differently; they couple to the interaction fields in different ways. In particular, 'right-handed' particle fields do not couple to the $W$ bosons at all.

So what does it mean to call a component of a field 'left handed'? The Dirac field can be thought of as the sum of two component fields, the left and right chiral components, which, in the zero mass limit, correspond to particles of definite and opposite helicity. The helicity of a particle is the projection of its spin in its direction of motion. Helicity eigenstates of a spin- $\frac{1}{2}$ particle involve the spin being either aligned or anti-aligned with the particle's direction of motion. These constitute two incongruent, 'handed' objects. By definition, left-handed massless particles are particles of negative helicity (their spin is opposite to their direction of motion), while right-handed particles are particles of positive helicity.

Helicity and chirality are not quite the same thing, however. A helicity eigenstate of a massive particle will involve both left- and right-handed pieces. Moreover, while the chirality of a particle is Lorentz invariant, the helicity of a massive particle is not. For example, one can Lorentz boost by a large enough velocity in the direction of the particle's motion so as to reverse that direction of motion

\footnotetext{
${ }^{24}$ I review some relevant details of the Weinberg-Salam theory in an appendix.
} 
while leaving the direction of spin unchanged. This cannot be done, of course, if the particle is massless and thus travelling at the speed of light. The helicity of a massless particle is an invariant property under the (restricted) Lorentz group.

A spinning object defines an axis: that about which it is spinning. For a given axis there are then two possibilities involved: if one looks along the axis of spin from a given direction, the object will either appear to be spinning clockwise or anticlockwise. These two possibilities are represented by associating each with a direction: the spin vector points along the axis of spin away from the point of view from which the spinning appears clockwise. This is equivalent to the definition of angular momentum vector $\mathbf{1}$ as $l^{i}=\varepsilon^{i j k} x^{j} p^{k}$ where $\varepsilon^{i j k}$ is completely antisymmetric in its three indices, $\varepsilon^{123}=+1$ and the components of $\mathbf{x}$ and $\mathbf{p}$ and given with respect to a conventional, right-handed set of Cartesian axes.

In the last analysis, in each case, the convention for associating a direction with a spinning object can only be specified via ostension. We can explain what we mean by left-handed and right-handed particles in terms of their relations to right-handed sets of axes, or in terms of their relations to typical clocks. But, if Bennett's Kantian hypothesis is correct, the meanings of these terms cannot be conveyed without ostension.

Turning now to the case of negative and positive helicity particles, one sees from the conventionality of the definition of the direction of spin, and from the need for ostension in specifying this direction, that to explain the difference between negative and positive helicity one must also ultimately appeal to ostension. The parallel with left and right hands is obvious. An intrinsic description true of a negative helicity particle will also be true of a positive helicity particle. Yet we can understand why there are two, incongruent, types in purely relational terms (i.e., in terms that do not presuppose an intrinsic difference). For two spin- $\frac{1}{2}$ particles of definite helicity travelling in the same direction they can be spinning in the same sense, or the opposite sense.

It turns out that the mathematics of parity violation does not involve treating being left-handed and being right-handed as different substantive and intrinsic properties. As is explained in the appendix, left and right components of the fields are distinguished in terms of their differing congruence relations to the righthanded coordinate systems with respect to which the theory is standardly written. One then goes on simply to assert of these two components that they interact differently.

This difference in the way they interact is not further explained in terms of different intrinsic properties possessed by the particles. Although I note in the appendix that the left and right components of the field are assigned different values of "weak hypercharge" and "weak isospin", these are not properties that explain their particular couplings to the gauge fields. For two varieties of particle to have the particular values of weak hypercharge that they have, for example, just is 
for them to couple to the gauge field $B_{\mu}$ with the relative strengths that they do. In fact, it seems plausible that, quite generally, particle varieties are only individuated in terms of their particular place in a network of differently interacting particles.

Moreover, it cannot be the helicity of a particle alone that determines which type of interactions it can undergo. The reason is that the 'left-handed' Dirac field component $\psi_{L}(x)$ - and its adjoint, $\overline{\psi_{L}}(x)$ - are associated with left-handed particles and right-handed antiparticles. (In the massless limit these correspond to negative and positive helicity eigenstates respectively.) Therefore the theory not only violates parity $P$, it also violates charge conjugation $C$, the interchange of particles with their antiparticles. However, the fragment of the theory described in the appendix is invariant under the combined transformation $C P$. Just as only lefthanded electrons and quarks couple to the $W$ s, only right-handed positrons and anti-quarks couple to them. ${ }^{25}$ So a description of the left-handed $\beta$-decay of a cobalt atom in intrinsic, relational terms will be equally true of a right-handed decay of an antimatter cobalt nucleus.

Does this fact, by itself, vindicate a relational account of handedness? Simon Saunders has suggested that it does (Saunders, 2000). His point is that the parityviolating law does not, after all, 'pick out' a particular handedness. Anything that is possible for a particle of one handedness is possible for particles of the opposite handedness, although it may only be possible for oppositely handed particles if they are also antiparticles of the first.

Just as the relationalist seeks to identify putatively distinct possible worlds containing nothing but single hands that supposedly differ solely in the sense of their handedness, Saunders urges that we should treat models of a parity-violating but $C P$-symmetric theory that are related by a global $C P$-transformation as different representations of the same state of affairs. If we describe a world in which the the first created process is a decay of a cobalt atom, there is no fact of the matter whether it was a decay of a matter nucleus emitting a right-handed antineutrino, or the decay of an antimatter nucleus emitting a left-handed neutrino. (Of course, if per impossibile the first created process of the actual world was a cobalt decay there would be a fact of the matter. But this would be a relational fact: did the decay involve the emission of a neutrino of the same handedness as particles that we in fact call "right-handed" and a particle of the same charge as particles that we in fact call "electrons"; or did it involve the emission of a neutrino of the same handedness as particles that we in fact call "left-handed" and a particle of the same charge as particles that we in fact call "positrons"?)

Unfortunately, $C P$ (or even $C P T$ ) symmetry does not by itself save the relation-

\footnotetext{
${ }^{25}$ The full Standard Model, involving all three generations of quarks, violates $P C$ symmetry. However, any theory based on a Lorentz-invariant Lagrangian $\mathcal{L}(x)$ formed from products of quantum fields at the point $x$ will be invariant under the combined transformation CPT.
} 
alist. Nor, indeed, is it even a necessary component of the relationalist's account. To see this, consider the following toy models. The first involves a possible world whose fundamental objects are hand-shaped and come in two varieties: "red" and "green". Red hands are never created, but they can "decay" into green hands. Now let us suppose that only red 'left' hands can decay into green 'left' hands. Red 'right' hands never decay and no green 'right' hands exist at all. This decay law clearly violates parity.

We can, however, extend our example so that it involves a $P$-violating but ' $C P$ ' symmetric law. In this second possible world, we now imagine that the red hands are 'charged' in that they attract or repel one another: similarly charged hands repel each other whereas oppositely charged hands attract each other. Now both left and right red hands can be 'negatively' and 'positively' charged. However, suppose that it is the case that only 'negatively' charged red left hands can decay into green (left) hands whereas 'positively' charged right hands can decay into green right hands. The law is now $C P$ symmetric: if a particular hand can decay, then so can its incongruent, charge-reversed counterpart.

Is this $C P$-symmetric law really more susceptible of a relationalist interpretation than the first, $P$-violating law? The problem that the relationalist still faces is to explain why negatively charged red right hands cannot decay into similarly oriented green hands when negatively charged red left hands (i.e., hands that are identical apart from the sense of their handedness) can. Relationalism denies that there is some intrinsic difference between the two types of hand that can ground and explain their different interactions. The fact that positively charged red right hands can decay into similarly oriented green hands does nothing to ameliorate the problem. Similarly, the relationalist is at a loss to explain why only left-handed electrons couple to $W$ bosons. How can it be that left-handed and right-handed electrons interact differently if the relationalist is correct in his deflationary account of what their being left- or right-handed consists in? The fact that right-handed positrons can couple to $W$ bosons is of little comfort.

There is another reason for being wary of invoking $C P$ (or CPT) symmetry to save a relational account of handedness: it fails to secure for the relationalist all that he desires. In his correspondence with Clarke, Leibniz, the arch-relationalist, insisted that two putatively distinct possible worlds differing solely over where the material universe was located in space were really just two ways of differently describing a single possibility. It is the homogeneity of space - that translations are a symmetry of Newtonian mechanics - that means that if one of these worlds is physically possible, then so is the other. Similarly, the relationalist will wish to see two models of a $P$-violating but $C P$-symmetric theory that are related by a global $C P$ transformation as but two ways of representing a single state of affairs.

What, though, should they say about the model one obtains from one model from such a pair by performing a global parity transformation without also inter- 
changing matter with antimatter? In the case of our second toy model, we obtain a world where it is negatively charged right hands and positively charged left hands that can decay. In the case of the a model of the Standard Model, we describe a world where right-handed electrons and left-handed positrons couple to $W$ bosons. The relationalist who sees the existence of a symmetry as a prerequisite of being able to identify the possibilities represented by models related by a non-trivial, global transformation must deny that these situations are equivalent to the original ones. He must even deny that they obey the same laws!

Something surely has gone wrong here. In what way does the parity-imaged model differ from the original? We call the quarks that couple to the $W$ s in the first model "left-handed", and call the quarks which couple to the $W_{\mathrm{s}}$ in the second model "right-handed", but what is the difference between them? In terms of the functional roles they play within the models, they are indistinguishable. If the original model was detailed enough to include human experimenters referring to left-handed quarks, then their counterparts in the second model will call the supposedly right-handed quarks "left-handed" too. The relationalist intuition is surely that the left-handed quarks of the first model should be identified with the so-called right-handed quarks of the second.

Despite the fact that the worlds described by the two models display a lawlike asymmetry between left and right, and despite the fact that the models are nominally the mirror images of each other, they should be regarded as solutions of a single theory, and the two models should be judged to describe a single possibility.

So let us now return to Earman's claim that the relationalist lacks the "analytical resources" to describe a law that can embrace both models. The relationalist must eschew terms such as "left" or "right", and he must not rely on a formulation that makes implicit use of such terms by relating the physics to, for example, righthanded coordinate systems. It seems, however, that the relationalist can indeed provide such a law.

Consider the first toy model again. The claim that all red hands which decay into green ones are handed in the same way embraces both the original possible world and its supposedly distinct parity-image. What is there to stop the relationalist claiming that this is a lawlike statement and, moreover, that it is primitive: it cannot be further explained in terms of more fundamental laws? Turning to the second, $C P$-symmetric, toy model, we can state that red hands that repel each other and which can decay into green hands are handed in the same way; red hands that attract each other and which can decay into green hands are handed in the opposite way.

Saunders offers a similar schematic law for $\beta$-decay:

PG Charge-conjugate $\beta$-decay processes are oppositely oriented.

He stresses that this a $P C$ invariant statement of $P$ violation. What is important for the present discussion is that it is also a $P$ invariant statement, even though it 
is an expression of $P$ violation. The statement is true both of a model where "left-handed" electrons couple to $W$ bosons and of a model where "right-handed" electrons do so. Of course, the law needs to be extended to include all varieties of fields - in particular, it must say something positive about the interactions which the electron-positron pair that cannot be produced in $\beta$-decays can undergo-but the outlines of how this is to be done are clear enough.

\section{Orientation Fields}

In characterizing the relationalist's position in this way, I am very close to Carl Hoefer. He writes: "The correct perspective, for either relationists or substantivalists, is this: $\mathrm{P}$-violating laws mandate that there shall be a certain, qualitative, spatial asymmetry in events. They do not explain the asymmetry or how it arises... Bringing in enantiomorphic objects allows one to 'anchor' the asymmetry descriptively, but is in no way explanatory of the asymmetry, nor do such objects become 'referred to' in the laws by being so used" (Hoefer, 2000: 253). In the case of the Standard Model, the enantiomorphic objects in question are actual left and right hands that are linked to the law, as we have seen, via the conventions that define what we mean by right-handed coordinate systems.

However, Hoefer also claims that parity violating laws are "purely phenomenological" and that this should, at least in part, ease any worries that we might have over the fact that the law-like asymmetry in phenomena is not ultimately explained. This seems to me to be contestable. The Standard Model is not a "purely" phenomenological law. There is a world of difference between the early descriptions of the weak interactions in terms of $V-A$ currents and the Standard Model together with the understanding that it provides us with of these truly phenomenological laws. The asymmetry in the phenomena is explained, albeit only in terms of a deeper asymmetry that is not. We postulate fundamentally handed particles (corresponding to massless spin- $\frac{1}{2}$ Dirac fields) and attribute different sets of interactions to oppositely handed fields. Why oppositely handed fields of the same particle type differ in this way is not explained. But there is no reason why the fact that they do should be seen as a phenomenological, rather than as a fundamental, fact.

Lack of a further explanation should not per se be seen as a problem for the relationalist. However, there is one feature of the relationalist's story that some will object to, and it is a feature that can lead back to substantivalism.

Recall that Van Cleve alleged that the problem faced by the relationalist was ineliminable reference to particulars rather than "action at a distance". We have seen that there is no ineliminable reference to particulars. However, the basic form of our proposed law - that all objects or processes of a certain relationally 
specifiable type are handed in the same way - is, in a certain specific sense, highly nonlocal. For the relationalist, being handed in the same way just is for two things to stand in certain spatio-temporal, and quite possibly spacelike, relations.

It is time to consider the recent 'reconstruction' of Earman's argument offered by Nick Huggett (Huggett, 2000). One of Huggett's central claims is that rather specific geometrical structures are involved in a proper formulation of parityviolating laws. He illustrates this claim with his own toy model quantum theory, involving two particles in one dimension, coupled by the following asymmetric potential:

$$
V\left(x_{1}, x_{2}\right)=\lambda\left(x_{1}-x_{2}\right)+\mu\left(x_{1}-x_{2}\right)^{2} .
$$

In this theory the two directions in its 1-dimensional space are not on a par. On measurement of their positions, the probability of finding particle 1 'to the left of' particle 2 (i.e., with a more negative position coordinate, relative to the coordinate expression of the potential given above) is greater than finding them the other way around. ${ }^{26}$

Huggett's claim is that the theory is not well-defined until an 'arrow of space' has been given, enabling us to say whether $x_{1}<x_{2}$ or $x_{2}<x_{1}$ 'in absolute terms, not just relative to some arbitrary coordinates' (Huggett, 2000: 233). He links this claim to the fact that the coordinate-free expression of the potential will involve the explicit introduction of an orientation field (in the case of the 1-dimensional theory, this field is simply a normalized 1-form). Similarly, if we were to express the equations of the Standard Model in a coordinate-free way, we would need to introduce an orientation field explicitly, and distinguish left- and right-handed components of fields by their relations to it, rather than to a standard coordinate system.

Huggett then puts the argument for substantivalism as follows, deliberately paraphrasing Earman's version of why the postulation of inertial structure to ground the distinction between absolute and relative motion licences the move to substantivalism:

... the 'absolutist' asserts that "the scientific treatment of motion ... requires some absolute quantities ... such as handedness. To make these quantities meaningful requires the use of an orientation, and this structure must be a property of or inhere in something distinct from bodies. The only plausible candidate for the role of supporting the nonrelational structures is the spacetime manifold." (Huggett, 2000: 236; cf. Earman, 1989: 125)

\footnotetext{
${ }^{26}$ Note that one unfortunate aspect of Huggett's example can be ignored. His theory violates both parity symmetry and permutation symmetry. It is not that one is more likely to find a particle of one type to the left of a particle of another type; rather one is more likely to find particle 1, that particular particle, to the left of particle 2 . The fact remains that it is solely the theory's violation of parity that requires the introduction of an orientation field when expressed in a coordinate-free way. Similarly the coordinate-free expression of the Standard Model requires the introduction of an orientation field, even though this theory does not violate permutation symmetry.
} 
The parallel drawn here is suggestive and worth pursuing. Pure inertial motion can be thought of as manifesting rather noteworthy non-local correlations. Assuming the correctness of Newtonian mechanics, one can, from the relative motions of just three force-free bodies, construct a spatio-temporal coordinate system with respect to which all three of these bodies are moving uniformly and in straight lines. ${ }^{27}$ This is already a highly non-trivial fact that one might feel calls for explanation, given that, as force-free bodies, the three bodies are supposed to be moving quite independently of each other. What is perhaps more striking is that every other force-free body is also moving inertially with respect to the coordinate system defined by the first three. The substantivalist offers a local explanation of these non-local correlations. According to him, the laws of motion constrain the motions of such bodies at each point of spacetime to be geodesics, as defined at each point by the affine connection.

Similarly, by reifying the orientation field, we can offer a local explanation of the non-local correlations between $\beta$-decays: that all neutrinos emitted in such decays are handed in the same way. According to Huggett's substantivalist, these correlations follow from the fact that the laws postulate that, at each point of spacetime, only quarks standing in one of the two possible relations to the orientation field at that point can couple to $W$ bosons.

If the introduction of a real orientation field provides a genuine and local explanation of the congruence of all $\beta$-decays at no cost we should surely admit such a field into our ontology. The question is, of course, whether the explanation is genuine, and whether there are costs. One might also wonder whether such a field supports spacetime substantivalism: does such a field obviously represent spacetime structure (as does the metric field of relativistic theories ${ }^{28}$ ) rather than just another real, physical field in spacetime?

Hoefer has objected to the move on different grounds. He holds that it amounts to nothing more than writing reference to particular bits of space into the laws:

It seems wrong for a law of nature to contain reference to a particular, contingent physical object. But it seems (to me) at least as wrong for a law of nature to contain reference to particular bits of space... That this is what is going on may be masked by talk of "absolute structures" or "a preferred $n$-form defined at all points", or something of this nature. But such terminology, while not literally incorrect, really only disguises the dependence on primitive identity to make the distinc-

\footnotetext{
${ }^{27}$ An elegant demonstration of this fact was given by Tait (Tait, 1883). For a nice account of it, see Barbour (1999: Chap. 6).

${ }^{28}$ This claim is, perhaps, controversial. Here I am siding with, e.g., Maudlin (1989: 318) and Hoefer (1998: 459-60) against Rovelli (1997: 193-4). For further discussion, see Pooley (2002: Chap. 4).
} 
tions between orientations for us. (Hoefer, 2000: 253)

The reader might be surprised at this assertion. Where, in the foregoing discussion of orientation fields, was reference made to particular bits of space? The reification of an orientation fields does not entail a commitment to primitive identity. Care is needed, however, as is illustrated by the following quite from Huggett, which follows his explicit introduction of an orientation field into his toy-model theory:

At this point it is worth noting for clarity that there is also a conventional aspect to such handed theories. For suppose the arrow of space now runs in the opposite sense; if $V_{A}$ remains the potential, then it will have the opposite handedness in space (compared to the original, or compared to some external bodies) and the system will behave differently. But if the potential also changes, $V_{A} \rightarrow-V_{A}$, then of course the dynamics will be as before. Thus, it does not make sense in this situation to ask in which direction the arrow of space runs, independently of a given Hamiltonian, and likewise it makes no sense to ask which sign of $V_{A}$ is correct, independently of an arbitrary choice of arrow. Thus the two possible arrows and two possible Hamiltonians only allow two distinct theories not four. This point acknowledged, we can talk of the arrow and the Hamiltonian and bear in mind the freedom this actually leaves. (Huggett, 2000: 234-5)

I agree with Huggett that there are certainly no more than two possible theories, not four. However, two readings of this passage are possible. And read in one way, precisely the wrong identifications are being advocated.

The orientation field is either supposed to be a real, physical field, or is supposed to represent some genuinely asymmetric structure of space or spacetime itself. If this is the case, then one cannot identify a theory that assigns a certain probability to the vector from particle 1 to particle 2 being aligned with the arrow defined by the orientation field, with a theory that assigns precisely that probability to the case where the two vectors are in the opposite alignment. Similarly, a theory that asserts that all electrons which are "congruent" to an orientation field couple to $W$ bosons and those which are "incongruent" do not, cannot be identified with a theory that predicts the same phenomena by asserting that all electrons which are incongruent to an orientation field couple to $W$ bosons.

However, the anti-haecceitist substantivalist who follows Hoefer in denying primitive identity relations between the spacetime points of different possible worlds will not be able to distinguish worlds that involve the same relations between orientation field and the matter fields, but that differ solely in terms of the relations that all of these fields bear to particular points of spacetime. But this is exactly 
the distinction that Huggett might appear to be upholding when he talks, for example, of the "arrow of space running in the opposite sense." It seems that we are here being asked explicitly to imagine the orientation field bearing a different relationship to particular points of space. ${ }^{29}$

So we can introduce an orientation field to ground a local explanation of the non-local asymmetries that the relationalist must postulate as brute, lawlike facts, in a way that doed not involve an implicit commitment to haecceitism and primitive identities. Nevertheless, it does appear to involve an unavoidable commitment to the reality of differences that are unobservable in principle: the theory that has only electrons "congruent" to such a field coupling to $W$ bosons and the theory that has only electrons "incongruent" to such a field coupling to $W$ bosons must be regarded as distinct theories, even though they are observationally indistinguishable. This problem is not merely the result of viewing the orientation field as a physical field that is distinct from spacetime. If one were to insist that it is spacetime itself that has an intrinsically asymmetric structure, and that the orientation field is just a mathematical device to encode such structure, one still would not be able to collapse the four formally distinct theories down to one. As Huggett notes (on the intended reading of the above quote), one is free to chose either of the the two possible orientation fields as encoding such structure. But this does not tell against there being a genuine metaphysical distinction between worlds where electrons bearing one type of relation to this structure interact in a certain way and worlds in which electrons bearing the opposite relation interact in an identical way. The only way to avoid postulating such a distinction is to adopt the relationalist's account of parity violation, together with its brute, lawlike, non-localities.

\section{Acknowledgements}

I am grateful to Harvey Brown, David Wallace, Mauricio Suarez, James Ladyman, Leah Henderson, Jeremy Butterfield, Carl Hoefer, Nick Huggett and Bill Child for comments on material for this paper and, particularly, to Simon Saunders for many discussions of handedness and parity violation. I would like to thank the editors for the invitation to contribute to this volume and to thank Katherine Brading especially for helpful and detailed comments on an earlier draft. Work for this paper was partly supported by the Arts and Humanities Research Board

\footnotetext{
${ }^{29}$ The alternative reading of Huggett's passage involves no such commitment to haecceitism. Instead one is merely noting that, relative to a fixed coordinate system, one can represent the asymmetric structure attributed to spacetime in two equal good ways. Although the coordinate system is kept fixed, one is contemplating a passive transformation that results in the orientation field of the mathematical model bearing a different relation to the physical orientation field (or to the asymmetric spacetime structure). Huggett has indicated that it was this freedom he was intending highlight (private communication).
} 
of the British Academy.

\section{A The weak interaction}

I should start by stressing that there are many reasons for thinking that the Standard Model is far from a final theory, not the least of which is the large number of unexplained parameters involved that must be fixed by hand for the theory to tally with experiment. There is also, as yet, no consensus on the correct way to extend the Standard Model so as to include neutrino mass. Indeed, this caveat is of relevance to our topic because some suggestions involve theories which are fundamentally parity symmetric. The standard electro-weak theory results from parity symmetry being spontaneously broken in such theories.

I propose to set these issues to one side. Treating the Weinberg-Salam theory as a fundamental theory poses the most severe challenge for any account of handedness according to which handedness is an extrinsic, rather than an intrinsic, matter. So if such an account can be defended in this context, a fortiori it should be defensible in others.

Central to the Standard Model are the left-handed and right-handed components of the various Dirac fields. ${ }^{30}$ Mathematically they are described in terms of the $\gamma$-matrices. In particular, for a Dirac field $\psi$, one has:

$$
\psi_{L}=\frac{1}{2}\left(1-\gamma^{5}\right) \psi, \quad \psi_{R}=\frac{1}{2}\left(1+\gamma^{5}\right) \psi
$$

where $\gamma^{5}=\gamma^{0} \gamma^{1} \gamma^{2} \gamma^{3}$. The $\gamma$-matrices might appear to be defined independently of our coordinate conventions: all that is required is that they obey the anti-commutation relations:

$$
\left\{\gamma^{\mu}, \gamma^{\nu}\right\}=2 g^{\mu \nu} I
$$

and $g^{\mu \nu}$ is left-right symmetric. However, they are tied to the coordinate system through the Dirac equation:

$$
\left(i \gamma^{\mu} \partial_{\mu}-m\right) \psi(x)=0
$$

If we reverse the sense of our coordinate system, say by relabelling the $x$ - and $y$ axes, we switch the roles of $\gamma^{1}$ and $\gamma^{2}$. Since, from eqn 3, these anti-commute, $\gamma^{5} \mapsto-\gamma^{5}$ and so the mathematical description of the left-handed component of a fermionic field with respect to a right-handed set of axes, is exactly the same description one gives to the

\footnotetext{
${ }^{30}$ In the following, I have drawn upon Peskin and Schroeder (1995) and unpublished lecture notes by I.T. Drummond and H. Osborn.
} 
right-handed component with respect to a left-handed set of axes. The standard mathematical descriptions of the chiral components of a fermionic field thus clearly relate them, more or less explicitly, to the the handedness of the Lorentz chart with respect to which the physics is formulated. There is nothing in the standard mathematical description of a left-handed field that is not equally suited, given different conventions, to describing a right-handed field. ${ }^{31}$

Having introduced the left-handed and right-handed components of the Dirac field, we can see how parity violation is implemented in the Weinberg-Salam theory. The theory is based on a Lagrangian density involving the Dirac lepton fields, the gauge fields and a scalar field, the Higgs field. The Lagrangian is invariant under certain local $S U(2) \times U(1)$ gauge transformations. The standard prescription followed in constructing a gauge field theory is to start with a Lagrangian that is invariant under some set of global gauge transformation and then create a locally gauge invariant theory by replacing the derivative operators in the original Lagrangian with "covariant derivatives" involving compensating gauge fields to ensure the required invariance. In this case, the original Lagrangian is the standard (parity-symmetric) Dirac Lagrangian for massless fields (for simplicity I only consider interactions involving electrons, positrons and their associated neutrinos):

$$
\mathcal{L}_{\text {kin }}(x)=\overline{\psi_{e}} i \gamma . \partial \psi_{e}+\overline{\psi_{\nu}} i \gamma . \partial \psi_{\nu} .
$$

No mass terms have been included because to construct a parity-violating gauge theory, symmetries under which the left and right chiral components transform differently must be gauged. This prohibits mass terms which mix left and right components and are thus not invariant under such transformations.

We now rewrite the Lagrangian as:

$$
\mathcal{L}(x)=\bar{L}(x) i \gamma . \partial L(x)+\bar{R}(x) i \gamma . \partial R(x) .
$$

where $L$ is a doublet involving the left-handed components of the neutrino and electron fields. Writing $\psi_{\nu}(x)=\nu(x)$ and $\psi_{e}(x)=e(x)$ :

$$
L(x)=\left(\begin{array}{c}
\nu_{L}(x) \\
e_{L}(x)
\end{array}\right)
$$

$R$ is a singlet involving the right-handed component of the electron field: $R(x)=$ $e_{R}(x){ }^{32}$

\footnotetext{
${ }^{31}$ I am indebted to David Wallace for this way of seeing how the handedness of field components is defined in terms of the handedness of the coordinate chart.

${ }^{32}$ Although I have not included the right-handed component of the neutrino field, the fact that neutrinos have mass means that the Standard Model must be extended to include it. The simplest way of doing so would be to include the field as a separate singlet term. However, as mentioned above, there are many rival proposals which experiment has yet to decide between.
} 
$\mathcal{L}$ is invariant under the $S U(2)$ transformations:

$$
L(x) \rightarrow e^{\frac{1}{2} i \alpha \cdot \tau} L(x), \quad \bar{L}(x) \rightarrow e^{-\frac{1}{2} i \alpha \cdot \tau} \bar{L}(x), \quad R(x) \rightarrow R(x),
$$

where $\tau$ are the $2 \times 2$ Pauli matrices. It is also invariant under independent $U(1)$ phase transformations of $L$ and $R$ separately. In particular, it is invariant under the separate phase transformations $L \rightarrow e^{-i \frac{1}{2} \chi} L$ and $R \rightarrow e^{-i \chi} R$ which may be written $\psi \rightarrow e^{i \chi} \gamma \psi$ where $Y=-\frac{1}{2}$ for $L$ and $Y=-1$ for $R$. These transformations are taken as the $U(1)$ transformations in an $S U(2) \times U(1)$ global symmetry group in order that the resulting gauge theory is invariant under local gauge transformations generated by the electric charge and electromagnetism is recovered.

A local $S U(2) \times U(1)$ transformation is then written:

$$
\psi \rightarrow e^{i \alpha(x) \cdot \mathbf{T}+i \chi(x) r} \psi(x)
$$

where $\mathbf{T}=\frac{1}{2} \tau$ acting on $L$ and $\mathbf{T}=\mathbf{0}$ acting on $R$. A gauge invariant Lagrangian is obtained by replacing the derivatives in eqn 6 with the covariant derivatives

$$
D_{\mu}=\partial_{\mu}-i g \mathbf{A}_{\mu}(x) . \mathbf{T}-i g^{\prime} B_{\mu}(x) \Upsilon .
$$

The result is:

$$
\mathcal{L}=\mathcal{L}_{\text {kin }}+g \bar{L} \gamma^{\mu} \frac{1}{2} \tau L . \mathbf{A}_{\mu}+g^{\prime}\left(\frac{1}{2} \bar{L} \gamma^{\mu} L+\bar{R} \gamma^{\mu} R\right) B_{\mu} .
$$

The consequences of gauging a symmetry group under which left and right field components transform differently are now manifest. Since $R$ is a singlet under the chosen $S U(2)$ transformations, it does not couple to the three component fields of $\mathbf{A}_{\mu}$ at all. That the left and right fields are assigned different values of "weak hypercharge" $Y$ means that the strengths of their coupling to the $U(1)$ gauge field $B_{\mu}$ is different.

The quantum field theory derived from this Lagrangian together with the appropriate Lagrangian for the free gauge fields is not yet empirically adequate because the four gauge fields all correspond to massless gauge bosons. It was known experimentally that there is only one massless gauge boson involved in electromagnetic and weak interactions, the photon. The solution is to exploit the mechanism of "spontaneous symmetry breaking". A scalar field with a gauge invariant potential term that has a minimum for non-zero values of the field is postulated. As a result of their coupling to this so-called Higgs field, all but one of the gauge fields acquire a mass and, with the right transformation properties under the gauge transformations assigned to the Higgs field, the massless field corresponds to the $U(1)$ gauge group of electromagnetism. The details need not concern us. All we 
need is that the 'physical' gauge fields corresponding to gauge bosons of definite mass are given by

$$
\begin{aligned}
W_{\mu} & =\frac{1}{\sqrt{2}}\left(A_{1 \mu}-i A_{2 \mu}\right), \\
z_{\mu} & =\cos \theta_{W} A_{3 \mu}-\sin \theta_{W} B_{\mu}, \\
A_{\mu} & =\sin \theta_{W} A_{3 \mu}-\cos \theta_{W} B_{\mu},
\end{aligned}
$$

where the Weinberg angle $\theta_{W}$ is defined by $\tan \theta_{W}=g^{\prime} / g$. Making the identification $e=g \sin \theta_{W}$, eqn 11 can be rewritten as:

$$
\mathcal{L}=\mathcal{L}_{\text {kin }}+\frac{g}{2 \sqrt{2}}\left(\mathcal{J}^{\mu} W_{\mu}+\mathcal{J}^{\mu \dagger} W_{\mu}^{\dagger}\right)+e_{e . m .}^{\mu} A_{\mu}+\frac{g}{2 \cos \theta_{W}} \mathcal{F}_{n}^{\mu} \mathcal{Z}_{\mu}
$$

where the weak, electromagnetic and weak neutral currents are defined as follows:

$$
\begin{aligned}
\mathcal{J}^{\mu}(x) & =\bar{L}(x) \gamma^{\mu}\left(\tau_{1}+i \tau_{2}\right) L(x)=\bar{\nu}(x) \gamma^{\mu}\left(1-\gamma_{5}\right) e(x) \\
j_{e . m .}^{\mu} & =\bar{L} \gamma^{\mu} \frac{1}{2}\left(\tau_{3}-1\right) L-\bar{R} \gamma^{\mu} R=-\bar{e} \gamma^{\mu} e \\
\mathcal{J}_{n}^{\mu} & =\bar{L} \gamma^{\mu}\left(\cos ^{2} \theta_{W} \tau_{3}+\sin ^{2} \theta_{W} 1\right) L-2 \sin ^{2} \theta_{W} \theta_{W} \bar{R} \gamma^{\mu} R \\
& =\frac{1}{2}\left[\bar{\nu} \gamma^{\mu}\left(1-\gamma_{5}\right) \nu-\bar{e} \gamma^{\mu}\left(1-\gamma_{5}-4 \sin ^{2} \theta_{W}\right) e\right] .
\end{aligned}
$$

Here again we see that only the left-handed fields couple to the $W$ bosons and that the coupling strengths of the left- and right-handed fields to the $Z$ are different. Only the left- and right-handed couplings of the electron/positron field to the photon $A$ is symmetric between left and right.

Let us now return to our original example of a parity-violating decay: the $\beta$-decay of cobalt atoms, $\mathrm{Co}^{60} \rightarrow \mathrm{Ni}^{60}+e^{-}+\bar{\nu}_{e}$. To model this in the electroweak theory outlined above, quarks need to be included. In addition to the doublet under local $S U(2)$ transformations, comprising the left-handed electron and electron-neutrino fields, there are weak isospin doublets containing the muon and muon-neutrino, the tau and tau-neutrino and three left-handed quark doublets. The first of these comprises the up quark field $u_{L}(x)$ and a linear combination of the down and strange quark fields $d_{\theta L}(x)=\left[\cos \theta_{c} d_{L}(x)+\sin \theta_{c} s_{L}(x)\right] . \theta_{c}$ is the socalled Cabibbo angle and its significance is not important for our discussion. The $\beta$-decay in question involves the decay of a neutron within the Cobalt nucleus into a proton with the emission of an electron and its antineutrino. This in turn is to be understood as the decay of a down quark bound within the neutron into an up quark $(n \sim u d d \rightarrow p \sim u u d)$. The interaction term responsible for this decay is $\mathcal{f}^{\mu \dagger} W_{\mu}^{\dagger}$ where $\mathcal{f}^{\mu \dagger}$ contains the term $2 \overline{e_{L}} \gamma^{\alpha} \nu_{e L}=\bar{e} \gamma^{\alpha}\left(1-\gamma_{5}\right) \nu_{e}$ as before and also $2 \cos \theta_{c} \overline{d_{L}} \gamma^{\alpha} u_{L}=\cos \theta_{c} \bar{d} \gamma^{\alpha}\left(1-\gamma_{5}\right) u$. The down quark decays into an up quark 
emitting a $W^{-}$boson which decays into an electron and its antineutrino. But since only left-handed quarks can couple to the $W$ boson, only left-handed quarks are involved in such decays and only left-handed electrons and positive helicity, right-handed antineutrinos are observed as a result.

\section{References}

Barbour, J.B. (1999). The End of Time: The Next Revolution in Our Understanding of the Universe. London: Weidenfeld \& Nicholson.

Barbour, J.B. and Bertotti, B. (1982). Mach's principle and the structure of dynamical theories. Proceedings of the Royal Society, London, A 382, 295-306.

Belot, G. and Earman, J. (2000). From metaphysics to physics. In From Physics to Philosophy, ed. J. Butterfield and C. Pagonis, pp. 166-86. Cambridge: Cambridge University Press.

Belot, G. and Earman, J. (2001). Pre-Socratic quantum gravity. In Physics Meets Philosophy at the Planck Scale, ed. C. Callender and N. Huggett, pp. 213-55. Cambridge: Cambridge University Press.

Bennett, J. (1970). The difference between right and left. American Philosophical Quarterly, 7, 175-91. Reprinted in Van Cleve and Frederick (1991: 97-130).

Brighouse, C. (1994). Spacetime and holes. In Proceedings of the 1994 Biennial Meeting of the Philosophy of Science Association, Vol. 1, ed. D. Hull, M. Forbes and R. Burian, pp. 117-25. East Lansing, MI: Philosophy of Science Association.

Brighouse, C. (1999). Incongruent counterparts and modal relationism. International Studies in Philosophy of Science, 13, 53-68.

Crawford, F.S., Cresti, M., Good, M.L., Gottstein, K., Lyman, E.M., Solmitz, F.T., Stevenson, M.L. and Ticko, H.K. (1957). Detection of parity nonconservation in $\Lambda$ decay. Physical Review, 108, 1102-3.

Earman, J. (1989). World Enough and Space-Time: Absolute versus Relational Theories of Space and Time. Cambridge, MA: MIT Press.

Frederick, R.E. (1991). Introduction to the argument of 1768. In The Philosophy of Right and Left, ed. J. Van Cleve and R.E. Frederick, pp. 1-14. Dordrecht: Kluwer.

Gardner, M. (1990). The New Ambidextrous Universe, revised edn. New York: W.H. Freeman and Company. 
Harper, W. (1991). Kant on incongruent counterparts. In The Philosophy of Right and Left, ed. J. Van Cleve and R.E. Frederick, pp. 263-313. Dordrecht: Kluwer.

Hoefer, C. (1996). The metaphysics of space-time substantivalism. Fournal of Philosophy, 93, 5-27.

Hoefer, C. (1998). Absolute versus relational spacetime: For better or worse, the debate goes on. British Journal for the Philosophy of Science, 49, 451-67.

Hoefer, C. (2000). Kant's hands and Earman's pions: Chirality arguments for substantival space. International Studies in the Philosophy of Science, 14, 237-56.

Huggett, N. (1999). Space from Zeno to Einstein: Classic Readings with a Contemporary Commentary. Cambridge, MA: MIT Press.

Huggett, N. (2000). Reflections on parity non-conservation. Philosophy of Science, 67, 219-41.

Kant, I. (1992 [1768]). Concerning the ultimate ground of the differentiation of directions in space. In The Cambridge Edition of the Works of Immanuel Kant: Theoretical Philosophy, 1755-1770, trans. and ed. D. Walford and R. Meerbote, 365-72. Cambridge: Cambridge University Press.

Kant, I. (1992 [1770]). On the form and principles of the sensible and the intelligible world. In The Cambridge Edition of the Works of Immanuel Kant: Theoretical Philosophy, 1755-1770, trans. and ed. D. Walford and R. Meerbote, 377-416. Cambridge: Cambridge University Press.

Maudlin, T. (1989). The essence of space-time. In Proceedings of the 1988 Biennial Meeting of the Philosophy of Science Association, Vol. 2, ed. A. Fine and J. Leplin, pp. 82-91. East Lansing, MI: Philosophy of Science Association.

Nerlich, G. (1994). The Shape of Space, 2nd edn. Cambridge: Cambridge University Press.

Peskin, M.E. and Schroeder, D.V. (1995). An Introduction to Quantum Field Theory. Cambridge, MA: Perseus Books.

Pooley, O. (2002). The reality of spacetime. D.Phil. thesis, University of Oxford.

Rovelli, C. (1997). Halfway through the woods: Contemporary research on space and time. In The Cosmos of Science, ed. J. Earman and J. Norton, pp. 180-223. Pittsburgh: University of Pittsburgh Press. 
Rynasiewicz, R.A. (1994). The lessons of the hole argument. British fournal for the Philosophy of Science, 45, 407-36.

Saunders, S.W. (2000). Incongruent counterparts; a Leibnizian approach. Manuscript.

Tait, P.G. (1883). Note on reference frames. Procceedings of the Royal Society, Edinburgh, Session 1883-4, pp. 743-5.

Van Cleve, J. (1991). Introduction to the arguments of 1770 and 1783. In The Philosophy of Right and Left, ed. J. Van Cleve and R.E. Frederick, pp. 15-26. Dordrecht: Kluwer.

Van Cleve, J. and Frederick, R.E., eds (1991). The Philosophy of Right and Left. Dordrecht: Kluwer.

Walker, R.C.S. (1978). Kant. London: Routledge and Kegan Paul. Pages 44-51 reprinted as "Incongruent Counterparts" in Van Cleve and Frederick (1991: 187-94).

Weyl, H. (1952). Symmetry. Princeton, NJ: Princeton University Press.

Wu, G-S., Ambler, E., Hayward, R.W., Hoppes, D.D. and Hudson, R.P. (1957). Experimental test of parity conservation in beta decay. Physical Review, 105, 1413-15. 